\title{
Substantially Higher Corrosion Resistance in AE42 Magnesium Alloy through Corrosion Layer Stabilization by ECAP Treatment
}

\begin{abstract}
P. MinÁRIK ${ }^{a, *}$, R. KRÁL ${ }^{a}$ AND B. HADZIMA ${ }^{b}$
${ }^{a}$ Department of Physics of Materials, Charles University, Ke Karlovu 5, CZ-12116 Prague 2, Czech Republic

${ }^{b}$ University of Žilina, Department of Materials Engineering, Žilina, Slovak Republic

The corrosion properties of magnesium alloy AE42, just extruded and with posterior eight passes through equal channel angular pressing after extrusion, were investigated in $0.1 \mathrm{M} \mathrm{NaCl}$ by electrochemical impedance spectroscopy. The obtained samples were compared using scanning electron microscopy micrographs of the corrosion layers created on the surface of the specimens. The background of the substantial higher corrosion resistance of the samples after equal channel angular pressing treatment was found to be the much thicker and stable corrosion layer and smaller separating fragments in comparison with the just extruded samples. The lowering of the grain size by severe plastic deformation, especially by the equal channel angular pressing treatment was found to be also responsible for the enhancement of the corrosion resistance in AE42 class of magnesium alloys.
\end{abstract}

PACS: $81.05 .-\mathrm{t}, 82.45 . \mathrm{Bb}$

\section{Introduction}

Magnesium alloys own several excellent properties which puts them in the focus of today's researchers. These properties include a low density, a high specific strength, good castability (suitable for high pressure die-casting), an ability to be turned/milled at high speed and a good weldability under controlled atmosphere. In addition, they have a relative low production cost, a high recycling ability and good damping properties. Nowadays even biodegrability and biocompatibility were found to be interesting properties in these materials. The main disadvantages are low elastic modulus, limited cold working and toughness, limited strength and creep resistance at elevated temperatures, high degree of shrinkage on solidification, high chemical reactivity and limited corrosion resistance [1].

Corrosion is in the most common way known as an electrochemical oxidation of metals in reaction with an oxidant such as oxygen. The speed of corrosion depends on many parameters such as composition of the metal and its surroundings, temperature, presence of radiation etc. In an electrochemical experiment $[2,3]$, the metal is immersed in a solution with an anode, a cathode and an ionic and an electrical current path between them two. The corrosion layer that appears on the surface is in some cases so strong that the electrical flow can be almost stopped. When such layer is stable under normal condi-

\footnotetext{
* corresponding author; e-mail: peter.minarik@mff.cuni.cz
}

tions, this is called passivation, and can be observed e.g. in aluminum or titanium in common environments. The composition and stability of the layer for magnesium dissolution has already been investigated with conclusions that magnesium dissolution in aqueous environment creates partially passive film $\mathrm{Mg}(\mathrm{OH})_{2}$ and/or hydride film $\mathrm{MgH}_{2}$. Stability of the hydroxide film depends on the $\mathrm{pH}$ of the solution. In the solution with the $\mathrm{pH}$ of 11 and higher, the film is stable [4], whereas when the $\mathrm{pH}$ is lower than 11 the film of $\mathrm{Mg}(\mathrm{OH})_{2}$ is only partially protective. However it was reported that the corrosion resistance of AZ31 magnesium alloy was enhanced by equal channel angular pressing (ECAP) treatment [5], which poses the question of the origin of this enhancement of corrosion resistance.

\section{Experimental procedures}

The examined magnesium alloy AE42 (4 wt\% of aluminum and 2 wt\% of rare earths, mishmetal) was prepared by hot extrusion at $T=350^{\circ} \mathrm{C}$ with an extrusion ratio $\mathrm{ER}=12$. Afterwards the specimens were machined to initial dimensions of $10 \mathrm{~mm} \times 10 \mathrm{~mm} \times 100 \mathrm{~mm}$. Both alloys were processed 8 times by ECAP to prepare the bulk material with fine grained structure, to a maximum equivalent strain of 8 [6], which means 8 passes (8P) following route $B_{\mathrm{c}}$ differently. Molybdenum disulphide grease was used as a lubricant. Table I shows manufacturing procedure of AE42-8P specimen. The angle $\Theta$ between two intersecting channels and the corner angle $\Psi$ were $90^{\circ}$ and $0^{\circ}$, respectively. 
TABLE I

ECAP procedure of AE42-8P specimens, $\mathrm{P}=$ pass.

\begin{tabular}{c|c|c|c|c}
\hline \hline \multirow{2}{*}{$\begin{array}{c}\text { Total count } \\
\text { of the routes }\end{array}$} & \multicolumn{4}{|c}{ Number of the route } \\
\cline { 2 - 5 } $8 \mathrm{P}$ & \begin{tabular}{c}
$210^{\circ} \mathrm{C}$, \\
\multirow{2}{*}{$8 \mathrm{~mm} / \mathrm{min}$}
\end{tabular} & $\begin{array}{c}195^{\circ} \mathrm{C}, \\
20 \mathrm{~mm} / \mathrm{min}\end{array}$ & $\begin{array}{c}185^{\circ} \mathrm{C}, \\
20 \mathrm{~mm} / \mathrm{min}\end{array}$ & $\begin{array}{c}180^{\circ} \mathrm{C}, \\
20 \mathrm{~mm} / \mathrm{min}\end{array}$
\end{tabular}

Electrochemical characteristics were investigated by electrochemical impedance spectroscopy (EIS) in $0.1 \mathrm{M}$ $\mathrm{NaCl}$ solution with the initial $\mathrm{pH}$ of 7 . The samples were mechanically polished by 1200 emery papers before each EIS tests. The characteristics were measured by a potentiostat/galvanostat Voltalab 10 (Radiometer-analytical SAS France) and a three electrodes connection (specimen, saturated calomel electrode and platinum electrode as working, reference and auxiliary electrode, respectively). In addition, a rotation of $1000 \mathrm{rpm}$ of the specimen was introduced for better homogeneity of the measurement. Measured data of the EIS tests were evaluated by EC-Lab V10 demo software (Bio-Logic SAS France). EIS tests were executed at room temperature from $100 \mathrm{kHz}$ to $20 \mathrm{MHz}$ with $10 \mathrm{mV}$ perturbation amplitude with respect to the open circuit potential (OCP). At least three measurements were performed for each sample. The details of EIS measurements are described elsewhere $[3,5]$.

The immersion was conducted in Hank's balanced salt solution with the composition presented as Table II.

TABLE II

Composition in $\mathrm{g} / \mathrm{l}$ of Hank's balanced salt solution.

\begin{tabular}{c|c|c|c|c|c}
\hline \hline $\mathrm{KCl}$ & $\mathrm{KH}_{2} \mathrm{PO}_{4}$ & $\mathrm{NaCl}$ & $\mathrm{Na}_{2} \mathrm{HPO}_{4}$ & $\mathrm{NaHCO}_{3}$ & D-glucose \\
\hline 0.40 & 0.06 & 8.00 & 0.0477 & 0.35 & 1.00
\end{tabular}

The microstructure of the corrosion layers was investigated by scanning electron microscopy (SEM). A SEM JEOL 50XA with a Bruker QUANTAX 200 energy dispersion microanalysis system (EDS) was used. He investigation was performed by detection of secondary electrons. Composition of studied area was identified according to X-radiation created after primary beam impact. The samples were embedded into the conductive resin, grinded by 1200 and 2400 emery paper, polished by $3 \mu \mathrm{m}$ and $1 \mu \mathrm{m}$ polishing disc and finally polished by OPS suspension.

\section{Results and discussion}

The microstructure of the initial extruded samples consisted of larger grains of $8-10 \mu \mathrm{m}$ and relatively fine grains of 3-4 $\mu \mathrm{m}$ around the large ones. The ECAP treatment resulted in grain refinement with grain size being 3-4 $\mu \mathrm{m}$, not shown here. The outcome of the EIS measurements, the Nyquist plots, of AE42 magnesium alloy are presented as Fig. 1 for both samples, after five minutes stabilization and also after seven days of immersion. The character of the graphs is alike for both samples and both durations, which means that the physical processes in the metal/solution interface are the same for both samples. After immersion of the specimen into the aqueous solution the surface is covered by a thick layer of magnesium hydroxide [7]. The plots after 5 min immersion are characterized by two well-defined capacitive loops, at high and medium frequencies, followed by an inductive loop in the low frequency region. The high frequency capacitive loop is related to the charge transfer resistance and the medium-frequency capacitive loop is connected with diffusion processes through the corrosion products layer. The inductive loop in the low frequency range is related to the relaxation of adsorbed species [3, 8-10].

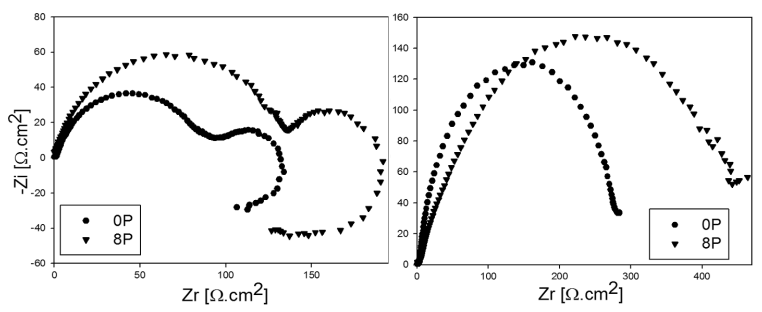

Fig. 1. Nyquist plots (a negative imaginary part of an impedance as a function of a real part) obtained by EIS measurements of samples just extruded and ECAPed after (a) five minutes of stabilization and (b) seven days of immersion.

The SEM micrographs presented in Fig. 2 are obtained after three days of immersion, because the layer was not clearly developed for lower immersion times, but it can be seen that the layer is created by islands of separated corrosion layers.

From the Nyquist diagram, the polarization resistance $R_{\mathrm{p}}$ can be evaluated by the "equivalent circuit method", which corresponds to the corrosion resistance of the specimen. For the analyses of the plots with two capacitive loops we used $R_{\mathrm{s}}-\left(R_{\mathrm{CT}} / Q_{\mathrm{DL}}\right)-\left(R_{\mathrm{F}}-Q_{\mathrm{F}}\right)$ equivalent circuit and for the analyses of the plots with one capacitive loop we used $R_{\mathrm{S}}-\left(R_{\mathrm{P}}-Q_{\mathrm{L}}\right)$ equivalent circuit (for details, see $[3,5,11])$. The total polarization resistance is equal to sum of the $R_{\mathrm{CT}}$ and $R_{\mathrm{F}}$ resistances or directly to the $R_{\mathrm{P}}$. The evaluated values of $R_{\mathrm{p}}$ for both types of specimens and both immersion times are presented as Table III.

TABLE III

Average values of $R_{\mathrm{p}}$ evaluated from Fig. 1 .

\begin{tabular}{c|c|c}
\hline \hline$R_{\mathrm{p}}\left[\Omega \mathrm{cm}^{2}\right]$ & $5 \mathrm{~min}$ & $168 \mathrm{~h}$ \\
\hline extruded & $80 \pm 5$ & $296 \pm 47$ \\
ECAPed & $121 \pm 11$ & $586 \pm 71$
\end{tabular}

The first noticeable fact is that the corrosion resistance of the ECAPed sample is almost double when compared to the only extruded one which corresponds to the wider loops in the Nyquist plots and which is more important in the micrographs of Fig. 2, where the layer created on ECAPed sample is almost twice as thick as of the just 

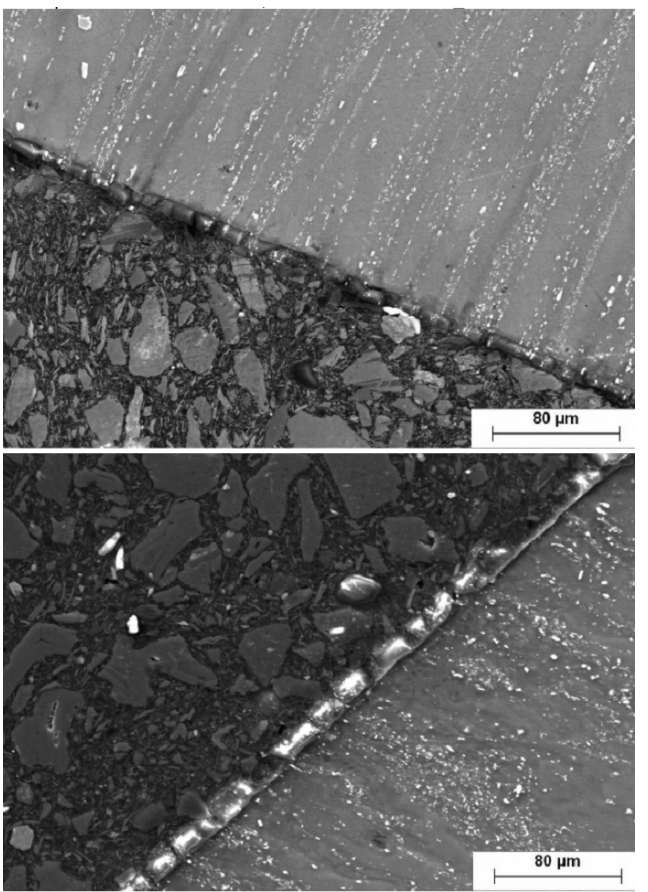

Fig. 2. Micrographs of the corrosion layer (SEM, backscattered electrons) after three days of immersion of just extruded (top panel, bulk upper right part) and ECAPed sample (bottom panel, bulk lower right part).

extruded one. As the corrosion layer acts as a resistor during the diffusion through the corrosion product layer, which is crucial action all through corrosion process, the thicker the corrosion layer, the higher corrosion resistance.

Figure 1 shows that the shape of the character of the Nyquist plots changed after seven days of immersion but again, the shape is the same for the both types of specimens. Now we are dealing just with one capacitive loop which corresponds to complex processes on the metal surface, including diffusion and charge transfer processes. A plot with only one capacitive loop results from an uneven thickness of corrosion products on the metals surfaces. This is proved by SEM micrograph, presented in Fig. 3, where solid corrosion layer can be identifiable on the metal/solution interface. The fact that the corrosion layer is evolving as a function of the immersion time was already shown in magnesium alloys before, e.g. [5 or 12], but an interesting fact is that the corrosion layer of the ECAPed sample is still much thicker when compared to the extrusion one. It also agrees with EIS measurements, which shows that the polarization resistance is again substantially higher, almost double in comparison with the extruded one.

Afterwards the long term immersion was conducted in order to investigate the next evolution of the corrosion layer. The SEM micrographs after thirty days of immersion are presented in Fig. 4 and it is quite visible that the corrosion layers of both specimens were further develop-
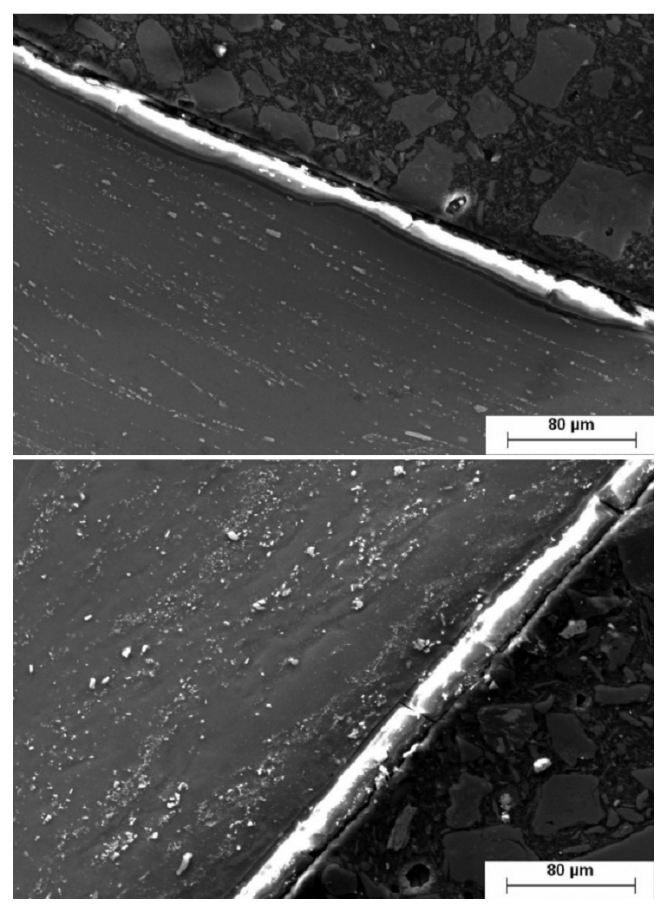

Fig. 3. Micrographs of the corrosion layer (SEM, backscattered electrons) after seven days of immersion of just extruded (top panel, bulk lower left part) and ECAPed sample (bottom panel, bulk upper left part).

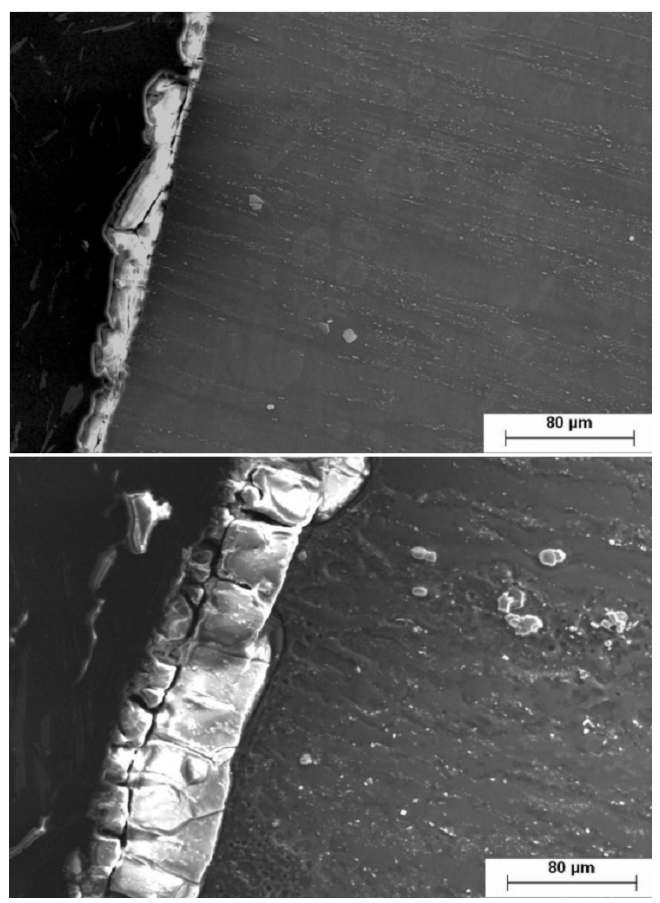

Fig. 4. Micrographs of the corrosion layer (SEM, backscattered electrons) after thirty days of immersion of just extruded (top panel, bulk right part) and ECAPed sample (bottom panel, bulk right part). 
ing. However, the difference between the thickness of the layer of the extruded specimen between seven and thirty days is much smaller as compared to the evolution of the layer on the ECAPed sample. The second important point is that there is difference in the fragments separating from the surface, much smaller from ECAPed sample: this is one of the mechanisms that stabilize the corrosion layer of the samples with the ECAP treatment [13].

\section{Conclusions}

EIS measurements were conducted on a AE42 magnesium alloy after extrusion only and extrusion followed by eight passes through the ECAP die. The resulting polarization resistance showed substantial higher corrosion resistance of the ECAPed samples. The background of such an improvement is much more stable and thicker corrosion layer created on the ECAPed samples. Though the composition of both alloys was the same, the ECAPed sample gained better corrosion resistance. Therefore, the severe plastic deformation is responsible for the enhanced corrosion resistance of AE42 magnesium alloy.

\section{Acknowledgments}

The authors gratefully acknowledge support by the grant SVV-2010-261307, SVV-2011-263307, support of The Czech Science Foundation under the grant 106/09/ 0482 and the support of the Charles University Grant Agency under the grant 355411/2011. This work has been supported by Scientific Grant Agency of Ministry of Education and Slovak Academy of Sciences, grant No. 1/0100/11 and No. 1/0262/10.

\section{References}

[1] B.L. Mordike, T. Ebert, Mater. Sci. Eng. A 302, 37 (2001).

[2] D.G. Enos, Tech. Report 33, 2 (1997).

[3] L. Škublová, Ph.D. thesis, University of Zilina, 2010.

[4] M. Pourbaix, Atlas of Electrochemical Equilibria in Aqueous Solutions, 2nd English ed., Tex.: National Association of Corrosion Engineers, Houston 1974.

[5] B. Hadzima, M. Janecek, M. Bukovina, R. Kral, Int. J. Mater. Res. 100, 1213 (2009).

[6] J. Zhao, Z. Wang, S. Sun, D. Zhao, L. Ren, W. Fu, J. Cent. South Univ. Technol. 16, 0349 (2009).

[7] G. Baril, G. Galicia, C. Deslouis, N. Pébère, B. Tribollet, V. Vivier, J. Electrochem. Soc. 154, C108 (2007).

[8] W. Liu, F. Cao, L. Chang, Z. Zhang, J. Zhang, Corros. Sci. 51, 1334 (2009).

[9] G. Baril, N. Pébère, Corr. Sci. 43, 471 (2011).

[10] G. Galicia, N. Pébère, B. Tribollet, V. Vivier, Corr. Sci. 51, 1789 (2009).

[11] L. Skublova, B. Hadzima, M. Bukovina, V. Skorik, J. Machine Manufact. 49, 18 (2009).

[12] B. Hadzima, M. Bukovina, P. Doležal, Mater. Eng./ Mater. Inž. 17, 14 (2010).

[13] B. Hadzima, M. Janeček, R.J. Hellmig, Y. Kutnyakova, Y. Estrin, Mater. Sci. Forum 503-504, 883 (2006). 